\section{NEUROLOSCIENCE}

\section{It's all in the details}

A rare variant of Epac2 that is found in some individuals with autism preferentially affects the basal over the apical dendrites of cortical neurons (PLOS Biol. 10, e1001350). The specificity of this effect suggests that the mutations found in people with autism may result in relatively subtle effects on brain architecture.

Epac2 is a brain-enriched guanine nucleotide exchange factor that regulates the function of the small GTPases Rap and Ras. Deepak Srivastava et al. found that knocking down Epac2 in cortical neurons in vitro reduced the size and complexity of their basal dendritic arbors. Overexpressing an autism-associated Epac2-coding variant had a similar effect. Mechanistically, this mutation disrupted the interaction between Epac2 and Ras. Indeed, inhibiting Ras also impaired basal-dendrite architecture.

As the number of identified rare coding variants associated with autism increases, it will be of interest to see whether they also result in phenotypes with an equal degree of specificity within a single cell. It will also be important to establish whether the autism-associated Epac2 mutation has a similar effect on neurons from other brain regions and whether there is a developmental window during which the impairment on basal-dendrite architecture can be reversed. $-J C L$

\section{NEUROSCIENCE}

\section{Restricted astrocytes}

Astrocytes are a type of glial cell and are essential for the maintenance of many neurological functions. Now, a new study (Science doi:10.1126/science.1222381) reveals that astrocytes are localized to specific domains in the central nervous system, with potential consequences for understanding region-specific brain functions.

Using reporter fate mapping in mice, HuiHsin Tsai et al. found that astrocytes migrate radially from the ventricular zone, forming distinct domains that are determined by their embryonic sites of origin in this zone. The astrocytes did not undergo secondary tangential migration later in development, in adulthood or after injury, suggesting that the restricted astrocyte domains remain stable.

The authors then investigated how astrocyte distribution influences interactions with neighboring neurons by selectively removing astrocytes from part of the ventral ventricular zone (the motor neuron progenitor domain). This

VIROLOGY

\title{
'Influenzing' the host response
}

A recent study identifies a new protein produced by the influenza $A$ virus (IAV) that can modulate the host response, thus providing insights into pathogenesis by this virus (Science 337, 199-204).

Segment 3 of the IAV genome encodes a single mRNA that encodes a subunit of the viral RNA-dependent RNA polymerase complex (PA), and Jagger et al. found that this viral segment contains an additional open reading frame (X-ORF) that can yield a different protein, PA-X, through ribosomal frameshifting. They showed that PA-X is composed of the endonuclease domain of PA and a C-terminal domain encoded by X-ORF and functions to repress the expression of cellular genes.

Jagger et al. then compared the pathology in mice caused by a reconstructed 1918 pandemic IAV that expressed PA-X with mutant IAVs that did not express PA-X. They found that the mutated viruses caused more severe disease than the wild-type 1918 pandemic virus, suggesting that PA-X reduces the pathogenicity of IAV. Moreover, infection with the mutated IAVs was associated with changes in host gene expression, including increased expression of genes involved in inflammation, apoptosis and T cell signaling compared with the wild-type IAV, as well as a reduced ability to induce host-cell shutoff.

This study contributes to our understanding of the roles of the virus and the host immune response in IAV pathogenesis, suggesting that immunopathology is a key aspect of IAVmediated disease.-MS

depletion altered the numbers of certain types of synapses, and astrocytes from adjacent regions could not migrate into the depleted area to rescue the synaptic dysfunction.

Thus, these results indicate that regional astrocyte allocation might be important for the development and maintenance of localized brain functions. The findings could also have implications for understanding brain regeneration, as they show that there are limitations in the astrocyte response to injury.-MS

\section{CANCER}

\section{Stromal protection from therapy}

Chemotherapy is the most common therapeutic option for metastatic breast cancer, but resistance readily arises. The link between metastasis and chemoresistance remains poorly understood. A recent report (Cell 150, 165-178) uncovers a signaling network linking metastatic and chemoresistant behavior and expands our understanding of the emerging role of the tumor microenvironment in controlling therapy responses.

Studying two related proteins previously associated with metastasis, CXCL1 and CXCL2 (CXCL1/2), Acharyya et al. find that they play a prometastatic part in cancer through their paracrine action on myeloid cells. Moreover, CXCL1/2-recruited myeloid cells can secrete factors that promote not only growth and metastasis but also the survival of cancer cells after chemotherapy.

Interestingly, the production of CXCL1/2 is upregulated in tumor cells treated with chemotherapy drugs. This stems from the proinflammatory effect of such therapies on normal tissues, leading stromal components to release cytokines that can upregulate the expression of CXCL1/2.

The authors find evidence that this signaling loop operates in humans with metastatic breast cancer and suggest that it may be a future target to prevent microenvironmental components from creating a protective milieu that allows the acquisition of therapy resistance.- $V A$

\section{REPRODUCTIVE BIOLOGY}

\section{Bringing up baby}

Whereas most expectant mothers love their gestating babies, their immune systems do not, instead seeing them as foreign bodies because of the paternal alloantigens they harbor. A new report (Ce// 150, 29-38) gives further insight into how maternal-fetal tolerance is maintained.

Peripherally-generated regulatory $\mathrm{T}\left(\mathrm{p} \mathrm{T}_{\text {reg }}\right)$ cells are believed to restrain immune responses 